\title{
«Si j'avais la vigueur de ces fameux latins...» Idéal et nostalgie du latin chez Théophile de Viau
}

\section{Melaine Folliard}

\section{CpenEdition}

\section{Journals}

Édition électronique

URL : http://journals.openedition.org/studifrancesi/7431

DOI : 10.4000/studifrancesi.7431

ISSN : 2421-5856

Éditeur

Rosenberg \& Sellier

\section{Édition imprimée}

Date de publication : 1 décembre 2009

Pagination : 481-505

ISSN : 0039-2944

\section{Référence électronique}

Melaine Folliard, « «Si j'avais la vigueur de ces fameux latins...» Idéal et nostalgie du latin chez

Théophile de Viau », Studi Francesi [En ligne], 159 (LIII | III) | 2009, mis en ligne le 30 novembre 2015, consulté le 08 janvier 2021. URL : http://journals.openedition.org/studifrancesi/7431 ; DOI : https:// doi.org/10.4000/studifrancesi.7431

\section{(c) (i) (9)}

Studi Francesi è distribuita con Licenza Creative Commons Attribuzione - Non commerciale - Non opere derivate 4.0 Internazionale. 


\section{"Si j’avais la vigueur de ces fameux latins...» Idéal et nostalgie du latin chez Théophile de Viau}

L'histoire a pris acte du lent déclin, de l'Antiquité tardive à nos jours, de la langue latine. Si, au commencement de l'âge moderne en Europe, elle cède progressivement le pas aux langues vernaculaires, elle continue de régner sur l'école, l'Église et la République des Lettres ${ }^{1}$. À travers l'essor de la prose en langue française au XVII ${ }^{\mathrm{e}}$ siècle ${ }^{2}$, le français prend l'ascendant sur le latin, tant du point de vue de la grammaire, de la rhétorique, que de la pensée dans l'invention et l'expression d'une littérature nationale. Le destin des deux langues semble définitivement scellé. À mesure que le siècle avance, le latin devient l'affaire des doctes, quand le français représente une norme curiale ${ }^{3}$.

L'œuvre de Théophile de Viau, publiée à partir de 1619, invite à s'interroger sur une telle séparation. Si le retentissement littéraire et mondain de l'auteur a consisté à explorer des genres nouveaux et à renouveler les formes esthétiques de la Renaissance par le medium linguistique du français, l'expression en langue latine, quoique paraissant réduite à la portion congrue chez Théophile de Viau, n'en est pas moins une constante dans cette œuvre singulière. De 1614 à 1626 en effet, de la première pièce imprimée du poète dans Le Violier des $M u s^{4} s^{4}$ jusqu'aux ultimes lettres manuscrites de l'exilé, le fait latin est prégnant. Est-ce en raison du succès inédit de la prose française à partir de 1620 , est-ce en raison de la fortune du «premier de nos grands prosateurs» ${ }^{5}$ et de l'éclat des postures du poète gaulois? Ces œuvres latines, qui pendant les siècles ultérieurs furent goûtées du public, ont longtemps été reléguées à leur inévitable singularité générique ${ }^{6}$.

(1) Fr. Waquet, Le latin ou l'empire d'un signe: $X V I^{e}-X X^{e}$ siècle, Paris, A. Michel, 1998, «L'évolution de l'humanité». Voir également le compte rendu qu'en fait A. LILTI, http://www.ehess.fr/centres/ grihl.

(2) Voir R. Zuber, Les «belles infidèles» et la formation du goût classique, Paris, Albin Michel, 1995 [1968], pp. 21-37.

(3) A. Viala, Naissance de l'écrivain: sociologie de la littérature à l'âge classique, Paris, Minuit, 1985.

(4) Epigramme liminaire intitulée In Gabrielis Roberti Opera, in Le Violier des Muses, Poitiers, Ch. Pignon et C. Courtoys, 1614.

(5) A. Adam, Théophile de Viau et la libre pensée française en 1620, Genève, Slatkine reprints, 2000 [1935], p. 88.

(6) La critique théophilienne s'est en effet attachée à l'analyse individuelle de ces trois textes en prose de Théophile. Voir L. Desjardins, Larissa, de Théophile de Viau, une fable milésienne du $17^{\text {e }}$ siècle. Etude littéraire, texte latin, traduction française, appendice bibliographique, "Humanistica Lova- niensia”, 19, 1970, pp. 471-484. La prose pamphlétaire du Theophilus in carcere a suscité davantage d'intérêt, en raison notamment de ses liens avec le procès de Théophile. Nous renvoyons au bilan bibliographique qu'en fait G. SABA dans Théophile de Viau, Paris-Rome, Memini, 2007, «Bibliographie des écrivains français», pp. 96 et 250, en y adjoignant l'étude de S. VAN DAMmE, L'épreuve libertine. Une bistoire culturelle du libertinage dans la France baroque, Paris, CNRS éditions, 2008. Le livre de G. SABA, Théophile de Viau: un poète rebelle, Paris, PUF, 1999, «Ecrivains», qui propose une analyse d'ensemble des lettres latines (pp. 202-211), rend compte, par sa disposition même, de la diversité constitutive de l'œuvre. Notre travail fera référence à l'édition de G. Saba, Théophile de Viau, Euvres complètes, Paris, Champion, 1999, «Sources Classiques», 18, 1999, 3 vol. et respectivement, t. I (Larissa), t. II (Theophilus in carcere), t. III (Lettres latines), où se trouve la traduction des textes latins par L. Desjardin, p. 179 sq. 
Car, si l'écriture latine concerne principalement des genres en prose chez Viau, c'est de textes divers, réalisés à différents moments de la carrière de l'auteur, qu'il s'agit: la brève nouvelle Larissa, publiée dans la première partie des Euvres en 1621, le Theophilus in carcere de 1624 écrit par un poète aux arrêts, les lettres latines enfin, qui pour beaucoup sont le fait du dernier Théophile, tout invite à saisir ces pièces dans la discontinuité formelle et idéologique d'une œuvre «rebelle».

Mais n'est-on pas en droit de penser, en raison de leur identité et de leur singularité linguistiques, que les pièces latines convergent vers une même intention esthétique? Il s'agira dans cette étude de s'interroger sur l'importance et l'incidence du fait latin dans la constitution d'une œuvre littéraire. Sous quelles conditions langue, culture et écriture latines permettent d'envisager à nouveaux frais la dynamique de la création chez Viau? En un mot, l'appréciation nostalgique de la langue témoigne-telle d'une ambition de l'auteur et d'un idéal de prose?

En raison du caractère multiple de ses usages et de ses appréciations, l'écriture en latin se contente-t-elle d'épouser les disparités génériques, stylistiques et linguistiques de la démarche littéraire de Viau, entérinant alors les ruptures chronologiques de l'œuvre, et rendant ainsi plus complexe la saisie d'un message esthétique? La production latine n'est-elle pas au contraire le lieu à partir duquel il est possible de reconsidérer les enjeux esthétiques, linguistiques et idéologiques de la création profane dans les années 1620 ?

Il importera dans un premier temps de rendre compte de la diversité de l'écriture en langue latine, représentative de l'œuvre protéiforme de Viau. Mais la pratique du latin, loin d'entériner les contradictions sur le plan de la langue, de l'invention et de la pensée de l'œuvre, atteste le retentissement singulier d'une culture chez Viau, bien au-delà de la lettre latine. Quel est le fondement d'une politique du latin chez Viau? En s'attachant enfin à en souligner l'incidence - langue, culture et écriture confondues - dans la constitution d'une figure de l'auteur, nous rappellerons que le fait latin, dans ses manifestations diverses, marque les jalons d'un idéal d'éloquence profane chez Viau.

\section{Des usages divers de l'écriture en langue latine chez Théophile de Viau}

Quoiqu'elles rappellent l'intérêt constant de l'auteur pour l'écriture latine, et qu'elles invitent à s'interroger sur les liens qu'entretiennent langue et prose, les pièces latines de Théophile de Viau n'en sont pas moins caractéristiques de la diversité de son œuvre.

\subsection{Diversité éditoriale}

Larissa, publiée en miroir avec la traduction du Phédon de Platon, parachève l'encadrement de la production poétique ${ }^{7}$. Cette pièce ressortit, autant qu'à l'ambition du polygraphe, à la stratégie éditoriale de la publication des Euvres d'un auteur jusqu'alors considéré, à travers ses publications collectives comme par ses multiples services de plume, pour ses talents poétiques. Le Theophilus, quant à lui, appartient au massif pamphlétaire que réalise Théophile en prison pour assurer sa propre défense. Ce texte circule de façon d'abord individuelle, avant d'être recueilli en 1625. Il sera intégré à la troisième partie des Euvres en $1626^{\circ}$. Le cas des lettres rappelle un

(7) G. SABA, OC, cit., t. I, Introduction, p. XXXV.

(8) Theophilus in carcere, s.l., s.n., 1624; Re- cueil de toutes les pieces faictes par Theophile, depuis sa prise iusques à present..., Paris, s.n., 1625; Les Euvres du Sieur Theophile, renenës, corrigées, 
autre aspect de la diffusion des œuvres de Théophile. Alors que les deux autres textes ont bénéficié d'une audience accrue, les lettres latines, manuscrites, s'adressent à un public en apparence restreint. Il faudra attendre l'édition que fournit Mairet en 1641 pour les voir rattachées au corpus des œuvres théophiliennes?. Cette situation particulière de l'édition posthume appelle deux remarques. Il s'agit d'une opération éditoriale entièrement dissociée des ambitions esthétiques et idéologiques de l'auteur: elle a lieu dans le contexte particulier des années 1640, alors que le genre épistolaire connaît un regain d'intérêt. La publication des Nouvelles Cuvres sert, autant que la mémoire de Théophile, les intérêts de fournit. Toutefois, la circulation manuscrite des lettres (latines et françaises) ne marque pas nécessairement chez Théophile le désaveu de l'imprimé. Plusieurs lettres rappellent que l'auteur ambitionne de faire éditer ses textes ${ }^{10}$. Poussé par la mode de l'épistolaire, le poète, qui a derrière lui une longue pratique du genre ${ }^{11}$, semble avoir tiré les leçons de la publication carcérale. La circulation manuscrite signale une prudence accrue de l'auteur depuis son procès.

\subsection{De multiples lecteurs}

Autant dire que ces textes aux destins divers visent une multiplicité de lecteurs. Public mondain, lecteurs parlementaires, adversaires idéologiques, protecteurs érudits, amis savants et confidents familiers, l'écriture en langue latine renvoie une image on ne peut plus variée des premiers lecteurs des œuvres de Théophile. Il ne faut certes pas les considérer de façon exclusive: la robe goûte la poésie en langue française ${ }^{12}$; la Cour a des lettres, et voit dans le latin un gage de légitimité culturelle. On est loin toutefois, dans le Theophilus, de l'évocation du plaisir mondain du lecteur, telle que la nouvelle Larissa la développe en 1621. Se rencontrent dans la pièce pamphlétaire de 1624 deux destinataires, que le poète entend placer aux antipodes l'un de l'autre: le jésuite Garasse, et les juges du poète. La disparité sociale des destinataires est accrue dans la production épistolaire. Elle vise, de près ou de loin, un public de familiers. Cela nous invite à reconsidérer, d'une part, les liens de Théophile au clientélisme - un Candale est plus qu'un protecteur ${ }^{13}-$ et, d'autre part, la nature du lien de parenté: un Paul de Viau est à la lisière de la relation sociale et privée. Véritable contrepoint au pathos de la pièce Lettre de Théophile à son frère qui clôturait l'édition de $1626^{14}$, cette épître finale tente de réguler la contradiction tragique des multiples appartenances de Théophile de Viau ${ }^{15}$.

Il importe de souligner combien ces lecteurs s'écrivent. La langue latine fait signe vers l'érudition parlementaire dans le Theophilus. En se coulant dans la langue

Ev augmentées..., Paris, Pierre Bilaine \& Iacques Quesnel, 1626.

(9) Nouvelles Euvres de feu Mr. Theophile, Composées d'excellentes Lettres françoises E latines..., Paris, Antoine de Sommaville, 1641.

(10) Voir par exemple OC, op. cit., t. III, Epistola XV, Ad Carolum Sanguinum, p. 104, et Epistola XX. Ad Principem Polonia, p. 109. Voir G. SABA, Théophile de Viau: un poète rebelle, cit., p. 204.

(11) Les Euvres de 1621 s'ouvrent sur une lettre à valeur préfacielle. Théophile est également l'auteur de l'Epître d'Actéon à Diane, que publie également Mairet en 1641. Beaucoup de lettres françaises font voir l'usage constant du genre sous une forme manuscrite depuis les débuts de la carrière du poète. Voir C. RizzA, Struttura et linguaggio delle "Lettres" di Théophile de Viau, in La prosa francese del primo Seicento. Ricerche e proposte, Cuneo, SASTE, 1977, pp. 45-87; et G. SABA, OC, cit., t. III, Introduction, p. XV sq. et, du même auteur, Théophile de Viau: un poète rebelle, cit., pp. 183-202.

(12) Le public parlementaire parisien goûte la poésie des recueils collectifs de poésie par exemple. Voir M. Bombart et G. Peureux, Politiques des recueils collectifs dans le premier XVII siècle. Émergence et diffusion d'une norme linguistique et sociale, in Actes du colloque international de Rennes, "Le Recueil littéraire”, Rennes, PUR, 2003, «Interférences», p. 240.

(13) Epistola XXII. Ad Dominum comitem de Candale, p. 110; Epistola XIII. Domino comiti de Candale, idem.

(14) OC, cit., t. II, pp. 238-247.

(15) Par le jeu par exemple de l'oxymoron: «Inimici gratia in meos ipse bostilem animum gererem», Epistola XXIV. Ad Paulum Fratrem charissimum, p. 111. 
des jésuites ${ }^{16}$, le prosateur fait voir combien Garasse, par les usages immodérés et impudiques de la prose française, s'en est détourné. Ecrire en latin place Théophile sous la tutelle des dévots, et surtout du Parlement. La prose carcérale tend vers ces deux destinataires; le latin est la langue de l'autorité ${ }^{17}$. C'est un type tout différent de lecteur qui est réinventé dans Larissa. La description d'un jeune noble sous les fers insiste, dès le commencement du récit, sur sa «naissance» ${ }^{18}$. La singularité sociale de ce personnage, sa délicatesse autant que sa connaissance des armes, son abattement initial autant que son endurcissement moral, l'évocation surtout de sa «beauté céleste» qui renaît de ses cendres, tout concourt à faire du personnage de Glison une incarnation moderne de la «supériorité» nobiliaire. Et qu'on le voit ainsi enlaidi par son asservissement au début du récit, favorise la «pitié» (misericordia) de la narratrice:

Dolebam ego vicem afflicti, et de Fortunae tam saeva varietate commiseratione illius moesta conquerebar... ${ }^{19}$

En réalité, c'est l'archétype de l'homme de cour qu'une plume sobre fait revivre:

...erat ingenium mite, placidi mores, sermo blandus, os amabile, et plane divinissimi vultus formosa et luculenta materia... ${ }^{20}$

En divisant le récit en deux tableaux, la narration attribue à Glison, puis à Larissa, deux servitudes différentes. La première, comme elle frappe la noblesse, est injuste dans ses causes; la seconde, métaphorique, n'est pas moins cruelle. Alors que Larissa volait au secours de l'infortuné jeune homme, celui-ci l'aide à quitter sa prison amoureuse dans la seconde partie. L'échange symbolique des servitudes peut ainsi être lu, dans l'économie de la narration, comme une tentative de l'auteur pour définir les conditions d'une «servitude volontaire» ${ }^{21}$. Fondée sur un déséquilibre originel (Larissa rappelle elle-même que son «obscure planète» s'incline devant la «supériorité naturelle» de son amant), elle tend, dans la fiction d'amour, à la reconnaissance mutuelle, et à la réalisation du plaisir. N'est-ce pas l'occasion pour Viau, au moment de sa consécration imprimée en 1621, de rappeler à ses nouveaux protecteurs combien le service de plume s'écrit dans l'expression d'un devoir mutuel et d'un service réciproque (beneficium) ${ }^{22}$ ?

\subsection{Variété générique}

Cette multiplicité de lecteurs que nous voyons consignés dans les productions latines explique pour une large part la variété générique et rhétorique qu'insuffle Viau à son œuvre. Et elle confirme, s'il en était besoin, la fréquentation assidue par le poète des modèles antiques et renaissants.

Il faut d'abord prendre acte de la variété des modèles dont dispose le prosateur latin: Apulée ou Pétrone pour le récit en prose; les grands orateurs antiques et néo-

(16) Fr. WAQUET, Le latin ou l'empire d'un signe, cit., p. 104.

(17) Ibid., p. 388.

(18) Larissa, OC, cit., t. I, p. 267.

(19) Idem.

(20) Ibid., p. 268.

(21) «Neque vero illius demum obire munera, ac laboribus meis otium illi comparare, sed et proprio servitio ultronem eius mancipium facta, socium colere et demereri conata sum», ibid., p. 267.

(22) «Intellexit tamen bene natus iuvenis quantum deberet bumanitati meae, et quoties beneficium accepit, puduit non potuisse referre, gratiasque verecundus egit iis verbis quibus solet urbanitas aulica trucioribus animis suppalpari», ibid., p. 268. 
latins pour l'éloquence judiciaire, parmi lesquels Cicéron ou Apulée occupent une place de choix ${ }^{23}$. La patristique ou les rhétoriques jésuites font aussi partie du bagage du polémiste. À côté de modèles attendus (Êrasme, ou encore le Pétrarque des Familiares), Théophile a en mémoire quand il écrit ses lettres le modèle linguistique de Scaliger ou la leçon de Lipse ${ }^{24}$. On ne reviendra ici que ponctuellement sur l'étendue de la culture rhétorique qui nourrit et donne forme à la prose latine de Théophile ${ }^{25}$. En raison même de sa diversité, il paraît difficile de parier sur l'homogénéité de l'écriture latine de Théophile: la rhétorique judiciaire, quand la vie de Théophile est devenue une affaire publique, s'impose; elle n'a que peu à voir avec l'éloquence du for privé, développée dans les lettres latines de façon singulière ${ }^{26}$. La prose latine est $a$ priori nourrie par des modèles rhétoriques disparates.

Les références latines sont variées. Mais, pour l'admirateur d'Horace, les auteurs latins sont avant tout des modèles de variété rhétorique, comme l'indique la nouvelle Larissa. Quoique n'étant pas la réécriture d'un passage particulier des Métamorpho$\operatorname{ses}^{27}$, la nouvelle n'est pas seulement un lointain souvenir d'Apulée. L'auteur ne se contente pas d'aller butiner chez Apulée, ou encore chez Pétrone ou Barclay, thèmes, motifs narratifs et figures de la fiction ${ }^{28}$. Il leur emprunte le dispositif énonciatif du récit enchâssé, fréquent dans les Métamorphoses, mais aussi, comme dans l'histoire de Psyché, le genre du «joli petit conte» narré par une vieille femme ${ }^{29}$. Sans donc considérer le patron apuléen de façon exclusive, Larissa fait voir combien, par la matière et la manière, Théophile hérite, en les condensant et les adaptant, les caractéristiques de la fable milésienne. Il condense le texte d'origine, tant la variété énonciative qu'on trouve dans l'Âne d'or se trouve intégrée à une courte séquence narrative. Le récit au passé, qui paraît comme détaché d'un corpus plus vaste, est interrompu brutalement par l'évocation du lieu et des conditions du récit. Cette multiplication des foyers narratifs facilite la superposition des voix et l'entremêlement des postures énonciatives. Viau adapte le texte source, puisque la vieille est moins édentée que chez Apulée, puisqu'un Glison semble moins effronté qu'un Thrasylle, puisqu'à la rudesse des rapports humains et du langage succède un idéal de tendresse et de sensualité linguistique. La variété doit donc être définie chez le prosateur latin comme l'exploration de modèles variés à partir desquels s'élabore la diversité énonciative. Il n'est pas anodin qu'à la même date paraisse la traduction du Phédon: le Traicté de l'immortalité de l'âme profite amplement du procédé d'enchâssement de la parole socratique dans un dialogue commémoratif de la philosophie ${ }^{30}$. Partagée entre imitation savante, et déjà

(23) C. Rizza a montré la dette du Theophilus, par le fond, la forme et le ton, envers l'Apologie d'Apulée (L'art de Théophile de Viau polémiste, in Actes de Marseille: Théophile de Viau, Actes du colloque du CMR 17, octobre 1990, R. DuCHÊNE éd., Paris-Seattle-Tübingen, PFSCL, 1991, pp. 146148).

(24) G. SABA, L'art épistolaire de Théophile de Viau, "Cahiers de l'Association internationale des études françaises", 39, mai 1987, p. 137.

(25) Nous insisterons donc peu sur l'héritage de la Renaissance française, d'autant que le travail a déjà été fait par J.-L. BAILLY, Modèles chez Théophile de prosateur, in Le Modèle à la Renaissance, C. Balavoine et alii éd., Paris, Vrin, 1986, pp. 141151.

(26) G. SABA, Théophile de Viau: un poète rebelle, cit., p. 206 sq.
(27) L. Desjardins, Larissa..., cit., pp. 471-484.

(28) Pour ne citer que quelques exemples: des Métamorphoses, l'auteur retient au moins le thème de la fille prisonnière ou, dans la description initiale, le motif des cheveux, métonymie de la beauté (Voir Les Métamorphoses ou l'Âne d'or, trad. O. SERS, Paris, Les Belles Lettres, 2007, «Classiques en poche», pp. 243 et 47). De Pétrone, le poète se souvient au moins de l'idéal de vraisemblance picturale que représente Apelle dans le Satiricon (du même traducteur, dans la même collection, 2001, p. 156). Il ne faut pas négliger les relais de la Renaissance, de Boccace à Marguerite de Navarre.

(29) Larissa, cit., p. 243.

(30) Voir notre étude Le Traicté de l'immortalité de l'âme... de Théophile de Viau, ou les voix du traducteur, à paraître dans la revue "Libertinage et philosophie". 
discrète, et liberté d'adaptation, la variété paraît être érigée en principe esthétique chez ce La Fontaine du premier XVII ${ }^{\mathrm{e}}$ siècle.

\subsection{La varietas: exercices et limites}

Or, est-ce pour s'engager davantage dans une esthétique de la variété que Théophile associe, dans un même mouvement d'écriture, les langues latines et françaises? C'est ce que donne à penser l'Apologie de Théophile, contemporaine du Theophilus ${ }^{31}$. Le latin y apparaît comme un bel émail qui vient rehausser la légitimité d'une défense de soi, et la dignité de la prose française. Soit le prosateur, en digne héritier des Essais, isole un vers qu'il adapte au contexte pamphlétaire. C'est alors une manière d'exclure d'une communauté de savoir son principal délateur. «Force gens de bien savent avec moi ce qui vous a piqué au jeu [...]» déclare le prosateur pour introduire des vers de l'Énéide qui témoigneront du mobile de Garasse:

\section{Manet alta mente repostum \\ Detectum crimen et laesae iniuria famae ${ }^{32}$.}

Le recours à l'érudition latine permet de faire le tri entre les lecteurs: «Mais laissons cela: cette vérité n'est pas encore bonne à dire». Et Garasse d'être abandonné à son esprit pesant.

Soit le travail est plus fin encore: la narratio incorpore alors sous la forme de citations des sources chéries par les différents destinataires du texte. Ainsi en évoquant le goût d'auteurs dont le seul nom fait autorité dans les lettres profanes ${ }^{33}$, ou lorsqu'un passage des Catilinaires est intégré à la péroraison («summum auxilium omnium gentium ${ }^{34}$ ), le texte entend se faire l'écho du goût et des valeurs parlementaires. L'Apologie dispose des lieux communs en langue latine, où se retrouve une communauté de savoir, de croyance et de culture: si l'évocation des Psaumes fait se rencontrer diverses catégories de lecteurs, la lettre même des modèles religieux favorise l'orientation polémique du texte. Le langage sera celui de l'apologétique: «in vanum laboraverunt gentes.... $>^{35}$.

Pour donner le change au jésuite, le prosateur n'hésite pas à prouver l'innocence de ses fictions poétiques par la casuistique d'un Vasquez:

Le jésuite Vasquez nous enseigne que les plus religieux peuvent avoir des pensées abominables qui ne sont pas fautes, d'autant que nous n'y persistons pas. Tu vero lector quisquis es falleris qui de simplicibus verbis mores nostros spectas feros, quidem ista obsident bonos praeterlabuntur. Les paroles sont paroles qui chez les casuistes ne sont pas plus, en cas d'offense, que les simples pensées ${ }^{36}$.

Il s'agit, au propre comme au figuré, de parler la langue jésuite, tout en reléguant certains pères trop zélés qui ont prêché publiquement contre Théophile dans le rôle des pharisiens. C'est Caïphe qui parle:

(31) C. RizzA lit le Theophilus comme un «raccourci» thématique et stylistique de l'Apologie (L'art de Théophile de Viau polémiste, cit., p. 146). La circulation d'une pièce française «accompagnée de son doublet latin» est fréquente depuis la Ligue: M. MARTIN, Conflits linguistiques et conscience nationale autour des états généraux de la Ligue de 1593 in Langues et identités culturelles dans l'Europe des $X V I^{e}$ et XVIII siècles, t. I, Nancy, Presses Universitai- res de Nancy, 2005, p. 332.

(32) G. SABA a montré que le premier vers, emprunté à l'Énéide (I, 26), est adapté à la situation personnelle de Viau, OC, cit., t. II, Notes, p. 363.

(33) OC, cit., t. II, Apologie de Théophile, p. 179 .

(34) CicÉron, Catilinaires, IV, 2.

(35) Psaumes, CXXVI, 1.

(36) Apologie de Théophile, p. 176. 


\section{Expedit unum hominem tantae invidiae reum mori pro populo ne tota gens pereat ${ }^{37}$.}

L'usage polémique du latin évangélique présente un double miroir de vérité. À un premier niveau de lecture, l'énonciation dénonce le recours trompeur au latin: c'est en profitant de «l'ignorance publique» que des accusations infondées ont été formulées contre la personne de Théophile. À un second niveau, en s'attachant à la lettre du texte, le polémiste prend le rôle du Christ, puis s'empresse de l'abandonner. La fiction de la langue latine délivre des «vérités occultes», accessibles à un nombre restreint de lecteurs:

Je sais que la prudence très accorte du Parlement tire du puits de Démocrite les vérités les plus occultes; qu'elle pénètre dans les obscurités plus ténébreuses où le mensonge et l'artifice se cachent; que c'est summum auxilium omium gentium, où l'innocence est assurée contre les efforts de l'envie et les ruses de l'imposture... ${ }^{38}$

Théophile joue encore sur la variété quand il fond en un même ensemble les deux langues. Le latin est parfois une mention ponctuelle, et érudite, dans l'argumentation en vernaculaire. Mentionner le spiritualis des platoniciens innocente, par la médiation de saint Augustin, le traducteur du Phédon. En estompant les écarts idéologiques entre auteurs profanes et chrétiens, Viau s'installe dans un régime de référence aux grands noms de la pensée ${ }^{39}$. Certains passages au contraire oublient de mentionner les emprunts. Le texte français est alors partiellement fécondé par la lecture de Virgile: «Pour votre regard, tout mon salut est de n'en espérer point ${ }^{40}$. D'autres passages, pour les besoins de la cause, convergent vers davantage de fidélité, sinon aux textes, du moins aux noms de référence: si «le sage Salomon et l'apôtre saint Paul» sont invoqués d'une commune voix pour innocenter l'auteur de la Première journée:

Le corps mortel, disent-ils, assomme l'âme et la traîne dans ses désirs charnels...,

c'est, à la fin de la période, saint Paul en personne qui vient défendre l'intégrité d'une âme chrétienne aux abois: faire ${ }^{41}$.

...et je fais le mal, dit S. Paul, que je ne veux pas faire et ne fais pas le bien que je veux

L'Apologie de Théophile invite à considérer comme un continuum idéologique et linguistique l'emploi mêlé du latin et du français.

Mais la fluctuation des deux langues ne laisse pas, dans un texte comme la Première journée, de poser problème. User simultanément du français et du latin contribue à une opposition qui excède les seuls critères linguistiques. Par les interventions du pédant Sydias, le texte français devient une bigarrure comique de latin macaroni$q^{42} e^{42}$ La langue sert ici un projet caricatural. Elle illustre en creux l'ambition esthétique de la nouveauté d'une prose française éloignée des «afféteries», autant qu'elle fait voir chez le locuteur, qui s'amuse de ce compagnon tout droit sorti des écoles,

(37) Ibid., p. 179. La citation provient de l'Évangile selon saint Jean (XI, 51 ).

(38) Ibid., p. 181.

(39) «Je n'entends point l'âme intellectuelle [...] mais j'entends cette âme, comme dit S. Augustin, susceptible des espèces corporelles, que les platoniciens ont nommée spiritualis», ibid., p. 177.
(40) Énéide, II, 354.

(41) Apologie de Théophile, p. 177.

(42) Voir J. RoYé, La littérature comique et la critique du latin, in Tous vos gens à latin. Le latin, langue savante, langue mondaine (XIV'-XVII siècles), E. Bury éd., Genève, Droz, 2005, «Travaux d'Humanisme et Renaissance, 405», pp. 223-236. 
un équilibre singulier entre connaissance des usages du monde et refus de l'asservissement idéologique. La pointe est latine, quand le narrateur, excédé par le babil du pédant, coupe court à une querelle sur les catégories de Porphyre:

Là-dessus, nous leur imposâmes silence et ordonnâmes que Sydias s'excuserait du démenti, et que l'autre tiendrait odor in pomo pour accident ${ }^{43}$.

La figure scolastique du pédant apparaît ainsi comme le premier jalon d'une défense et illustration de la liberté de parole. En un mot, la langue latine cristallise tout ce que la première personne n'est pas dans ce récit des valeurs mondaines.

Trois catégories de problèmes s'imposent à la lecture d'une pratique du latin chez Viau. Le premier est bien évidemment d'ordre linguistique. Les emplois du latin semblent soumis, selon les genres et les moments de l'œuvre, à des appréciations diverses, qui confinent à la contradiction. En appeler à l'autorité de la langue latine en 1624, c'est précisément ce que paraît vouloir récuser un an plus tôt celui qui s'était fait le chantre d'un «langage nouveau»: car le latin, en comparaison au français, tend à représenter l'inique autorité contre laquelle la littérature profane s'invente. L'emploi du latin à l'échelle de l'œuvre de Viau interroge ses options linguistiques à partir de 1620. Si, bon gré mal gré, l'auteur s'inscrit dans l'impétueux mouvement de la normalisation linguistique ${ }^{44}$, Viau manifeste à plusieurs reprises une forme d'opposition à la montée en puissance du clan de Malherbe. La langue latine est-elle le terrain où va pouvoir se manifester le plus distinctement une pensée linguistique singulière? Favorise-t-elle la construction d'une identité de l'auteur, en faisant correspondre - l'écriture française ne le permet pas - théorie et pratique? Les orientations linguistiques sont indissociables des options esthétiques. Quoique décriant régulièrement «l'antiquaille» et ses illusions, le prosateur, ou le poète français, ne cherche pas à en proscrire l'usage $^{45}$. C'est la raison pour laquelle les connotations changeantes prêtées à la langue latine s'inscrivent chez Viau dans une relation paradoxale à la culture littéraire de son temps. L'écriture latine permet, en d'autres termes, d'interroger à nouveaux frais le problème de l'imitation. On ne saurait enfin distinguer les questions linguistiques et esthétiques de la discontinuité idéologique de l'œuvre de Viau. La Première journée comme l'Apologie conviennent d'un latin de simulacre. Mais est-il possible de revendiquer en 1624 une lecture machiavélienne de l'abus populaire, comme dans le récit de cette possédée qui, par quelques mots de latin mal prononcés, rend plus crédible l'existence du diable? Pour le dire autrement, selon quelles conditions est-il tenable d'alléguer, au cours d'une brève carrière d'écrivain, saint Paul, saint Augustin et la galerie des Pères et des apologètes, tout en ménageant une place de choix à Apulée ou Pétrone, connus d'abord à cette date pour leurs impudicités? Pour tenter de réduire ces tensions, il faut, en prenant du champ, évaluer le retentissement de la culture latine dans l'œuvre de Viau.

(43) Première journée, OC, cit., II, p. 19.

(44) D. Trudeau, Les inventeurs du bon usage, Paris, Minuit, 1992, p. 141 sq.

(45) Voir C. Rizza, Place et fonction de la my- thologie dans l'univers poétique de Théophile de Viau, in La Mythologie au XVII siècle, Colloques du C.M.R. 17, janvier 1981, L. GODARD DE DONVILle éd., Marseille, 1982, pp. 255-264. 


\section{Retentissement de la culture latine: béritages et postures}

\subsection{Les conditions d'un savoir latin: «cursus» et lignage}

Les frontières sont poreuses d'une langue à une autre. Mais peut-il en être autrement en 1620? Le français paraît vouloir se désolidariser de son substrat latin ${ }^{46}$. Mais c'est souvent un effet de trompe-l'œil: les pratiques linguistiques et esthétiques restent fortement médiatisées par la langue latine ${ }^{47}$. On ne devra pas confondre l'essor d'une culture nationale et son expression littéraire ${ }^{48}$, les cadres de la mondanité littéraire et les options politiques et esthétiques qu'elle entraîne dans l'écriture française, avec la persistance d'une culture humaniste dont le latin reste l'expression. Montaigne avait montré en son temps que le goût et l'exercice du latin étaient indissociables d'une bonne éducation ${ }^{49}$. Si les lettres profanes, publics nouveaux et enjeux politiques obligent, semblent privilégier une esthétique du français, on ne devra pas négliger le rôle capital que continue à jouer le latin dans l'univers du savoir. L'imprimé parisien témoigne du rôle fondamental du latin dans l'appréhension de l'idée même de connaissance $^{50}$. Selon J.-W. Binns, jusqu'en 1640 «toutes les formes élevées du savoir, de la littérature au droit, des sciences à la théologie $\rangle^{51}$ passent par ce medium linguistique.

Il ne faudra donc pas s'étonner que la langue latine ait une place de premier choix dans la formation intellectuelle de Théophile. Si les œuvres n'offrent que peu de prise à une analyse documentaire de ce type, le fait latin atteste toutefois la solide culture humaniste de Viau ${ }^{52}$. La pédagogie protestante semble avoir laissé des traces dans le plurilinguisme revendiqué par l'auteur. Loin d'être le fruit d'une inspiration diabolique $^{53}$, le latin est une compétence linguistique parmi d'autres:

[La possédée d'Agen] grommel[ait] quelques mots de latin mal prononcé. Je lui parlai latin le plus distinctement qu'il m'était possible, mais je ne vis jamais aucune apparence qu'elle l'entendît; je lui dis du grec, de l'anglais, de l'espagnol et de l'italien, mais à tout cela ce diable ne trouva jamais à répondre un son articulé ${ }^{54}$.

Que la langue soit un instrument de communication, de savoir-vivre et de jugement participe, dans cette fiction, à la valorisation discrète d'un ethos protestant. Elle reflète l'idéal éducatif luthérien où «les langues sont les fourreaux qui renferment l'esprit». ${ }^{55}$ Cette valorisation du don des langues correspond très certainement aussi

(46) Voir l'analyse grammaticale de N. FouRnIER, Outillage et bricolage dans la grammaire française aux XVI et XVII siècles: l'exemple de "La grammaire et syntaxe françoise” de Charles Maupas ([1607] 1618), in Langues et identités culturelles dans l'Europe des XVI et XVII siècles, cit., pp. 25-39.

(47) Le «latino-centrisme» constitue autant de points de résistance à ce «grand mouvement de promotion de la langue française» dans lequel s'inscrit la grammaire de Maupas. L'«autonomie et précellence du français» est loin d'être théoriquement effective, ibid., pp. 38-39 et 27.

(48) Voir H.-J. Martin, Livre, pouvoirs et société à Paris au XVII siècle, Paris, Droz, 1999 [1969], t. I, pp. 25-27.

(49) De l'institution des enfans, I, XXV, in Les Essais, J. Balsamo-M. Magnien-C. Magnien-SimoNIN éd., Paris, Gallimard, 2007, «Bibliothèque de la Pléiade», p. 180.

(50) Voir H.-J. Martin, Livre, pouvoirs et société à Paris au XVII siècle, cit.
(51) J.-W. Binns, cité par Fr. Waquet, Le Latin ou l'empire d'un signe, cit., p. 105.

(52) G. Saba souligne l'importance de l'héritage scolaire des manuels de rhétorique dans la composition des lettres latines (Théophile de Viau: un poète rebelle, cit., p. 205).

(53) Comme ses détracteurs le laissent à penser: «Ils pensent que le vin soit le feu qui m'inspire/Cette facilité dont tu me vois écrire, /Et qu'on ne me saurait ouïr parler latin/ Si ce n'est que je sois à la Pomme de Pin...» (Lettre de Théophile à son ami Tircis, OC, cit., t. II, v. 85-88, p. 145).

(54) OC, cit., Première journée, p. 18.

(55) «Vaginarum vice sunt linguœe in quibus gladius ille Spiritus...» déclare Luther, «très sévère pour l'ancienne éducation», dans son Colloquiorum scholasticorum libri quatuor, Genève, 1563, cité par G. COMPAYRÉ, Histoire critique des doctrines de l'éducation en France depuis le XVI siècle, Paris, Hachette et Cie, 1883, $4^{\mathrm{e}}$ édition, t. I, p. 437. 
à des compétences réelles chez ce huguenot de Théophile, dont le cursus scolaire et universitaire, des Collèges de Nérac et Montauban jusqu'à l'université de Leyde, en passant par l'Académie de Saumur, a principalement eu lieu en latin. Le caractère chaotique des années de formation de Viau rappelle à l'aune de quels aléas sociaux Théophile s'est engagé dans le service de plume. Mais l'étude de la médecine, à Bordeaux ou à Leyde, n'a fait que renforcer une capacité linguistique. Un seul exemple servira à montrer que les réseaux humanistes ont orienté les lectures de Viau. Lipse, dont l'Epistolica institutio paraît être un modèle de référence pour l'épistolier latin ${ }^{56}$, a probablement été découvert et commenté lors du séjour en Hollande ${ }^{57}$. L'inscription de Viau dans un cursus protestant confirme donc la prééminence d'un savoir latin.

Ce savoir est lié à la revendication d'un lignage, où l'exercice par les aïeux de prestigieuses charges sociales cède le pas, d'une génération à une autre, à l'amour généreux du père pour les lettres humanistes: «scito mibi Avum fuisse Reginae Navarraeorum a secretis, patrem a teneris annis quibus decuit sumptibus literis bumanioribus incubuisse... ${ }^{58}$. C'est la raison pour laquelle il serait abusif de faire de la langue latine le signe d'une appartenance protestante. La lettre latine que Théophile adresse à son frère pour l'enjoindre de se convertir paraît même exprimer l'idée opposée: le locuteur prend plutôt la pose du prosélyte, dans le vocabulaire éprouvé de l'apologétique catholique ${ }^{59}$. Mais le recours au latin ici ne favorise pas tant l'expression d'une rupture religieuse qu'il ne cherche à rappeler un sentiment d'appartenance familiale. À s'en tenir à l'aspect documentaire et communicationnel du texte, Théophile rappelle que l'espace d'échange est fondé à l'origine sur une éducation et une coutume communes, même s'il s'agit de s'en défaire: «Non tua ista, si bene nosti, sed nutricis tuae religio, et praeceptorum qui te magis de consuetudine quam de propria ipsorum libidine fortassis educarunt $\rangle^{60}$. Surtout, telle qu'elle est composée, la lettre consacre la légitimité du lien de parenté, par-delà les divisions sociales ou religieuses («Verum de regiis et divinis hactenus» $\left.{ }^{61}\right)$. L'expression en langue latine assure un équilibre entre l'bumilitas des origines provinciales et la dignité d'une culture familiale. Le latin permet de reconquérir une intégrité sociale. Dans le Theophilus, le «fonds» clairacais est certes modeste, il n'en est pas moins le signe de l'hospitalité des valeurs curiales, n'en déplaise à Garasse: «Eam domum quam tu Cauponam vocas, Aulici plures atque ii melioris notae dignati sunt invisere, et pro tenui nostro proventu aliquot dies frugaliter excepti saltem immunes abiere» ${ }^{62}$. Théophile se fait l'ombre de ses illustres destinataires parisiens ${ }^{63}$. Le latin renvoie, à travers l'échange prosaïque d'une lettre, à une communauté de biens: «noster Daniel [le frère qui cultive les terres] [...] fundum nostrum [...]», la plasticité du pronom possessif fait insensiblement évoluer le texte d'une opposition communautaire vers sa sublimation dans la communion familiale. $\grave{A}$ travers l'échange en latin a lieu un partage de compétences sociales non dénué d'esprit, puisque le frère, lui aussi, manie la plume aussi bien que l'épée: «sed illud opus [la réponse à la lettre] ni taedat vasconicis versibus confice» ${ }^{64}$.

(56) G. SABA, L'art épistolaire de Théophile de Viau, cit., p. 139.

(57) G. COHEN rappelle l'importance de l'enseignement en latin à l'Université de Leyde, l'audience dont y a bénéficié Juste Lipse, mais aussi, dès la fondation de l'Université, la vénération qu'on voue à ce «divinus [...] Plato», Écrivains français en Hollande dans la première moitié du XVII siècle, Paris, Honoré Champion, 1920 , p. 147 sq. Il va sans dire que le latin commémore cette époque, quand Théophile échange en 1623 avec son ancien maître hollandais Bertius (Epistola X et XI, OC, cit., t. III, pp. 102-103).
(58) Theophilus in carcere, cit., p. 163.

(59) Voir par exemple les métaphores du texte: «tenuior est haereseos nebula quam ut remoraripossit audacter intuentem», Epistola XXIV, cit., p. 112.

(60) Ibid., p. 112.

(61) Idem.

(62) Théophilus in carcere, cit., p. 163.

(63) «Sunt enim plerique omnes praeclaro genere orti, et quibus iampridem res familiaris Maioribus suis ampla fortuna fortunae securos facit...», ibid., p. 162.

(64) Epistola XXIV, cit., p. 112. 


\subsection{Une œuvre en référence à la culture latine}

L'éclectique bibliothèque latine de Théophile rend certes compte du foisonnement tout humaniste des lectures, érudites ou mondaines, profanes ou sacrées dont les Euvres ont fait leur matière. Mais elle ne doit pas manquer de rappeler la profonde cohérence d'une érudition latine pleinement assumée. Rappelons d'abord à quel point l'œuvre française paraît s'être enrichie des modèles latins. C'est le cas de la prose. Comment comprendre les prises de positions esthétiques de la Première journée, sans rappeler la dette de Théophile envers le Cicéronien d'Érasme ${ }^{65}$ ? Par ailleurs, Ovide est fréquemment convoqué dans l'énonciation érotique ou épidictique, mais aussi dans la prose, latine ou française. Des épîtres en vers réunies par Billaine et Quesnel en $1621^{66}$ jusqu'à l'Epître d'Actéon à Diane, faite «à l'imitation d'Ovide» selon les mots de Mairet, tout rappelle l'immense succès éditorial de Nason ${ }^{67}$ dans lequel s'est fondu le poète, le prosateur ou le dramaturge ${ }^{68}$. Ovide constitue un réservoir de genres et de postures. Mais on ne saurait réduire l'inspiration poétique latine de Théophile à un échange fructueux avec Nason. Le chantre de la variété moderne fait aussi appel, par le fond et la forme dans l'élégie, aux modèles intermédiaires de Catulle, Tibulle ou Properce ${ }^{69}$; Virgile bien sûr, mais Juvénal, Perse ou Lucrèce ne sont pas en reste, des Satires au Traicté de l'immortalité de l'âme. L'œuvre crée ainsi une référence accrue à la culture latine, preuve éclatante d'un dialogue permanent avec les modèles antiques. C'est un dialogue critique qu'entretient l'énonciation, comme dans le traitement épidictique de l'exil ovidien. L'impertinence légendaire de Nason souligne le dévouement de Théophile à son auguste de roi:
... Ovide ne fut chassé
Que pour avoir osé médire.
Moi, dont l'esprit mieux arrêté
D'une si sotte liberté
Ne se trouva jamais capable.$^{70}$

Le dialogue est savant dans la paraphrase d'une ode horatienne ${ }^{71}$ : la médiation de l'antique allie une esthétique de la suavitas à une éthique de la singularité.

(65) Le désir d'une écriture à la moderne et la récusation de l'imitation dans le chapitre premier de la Première journée semble en effet se calquer sur la critique du Cicéronien: «Je dois parler à des chrétiens de la religion chrétienne. Est-ce que d'aventure, pour tenir un discours adapté, je vais m'imaginer que je vis à l'époque de Cicéron [...]? Ne sera-t-il pas bien fade l'orateur qui aura cousu à son thème ces sortes de haillons empruntés à $\mathrm{Ci}$ céron?», in Erasme, Colloques, D. MÉNAGER trad., Robert Laffont, 1992, «Bouquins», p. 428.

(66) Voir G. SABA, L'Épître d'Actéon à Diane de Théophile de Viau: lettre hérö̈que et/ou nouvelle psychologique à la première personne, in Ouverture et dialogue, Mélanges offerts à W. Leiner, Tübingen, Narr, 1988, pp. 395-403.

(67) Voir H. Lamarque, L'édition des Euvres d'Ovide dans la Renaissance française, in Ovide en France dans la Renaissance, CECNL, 1, Toulouse, 1981, pp. 13-40. A l'échelle du XVII siècle, Ovide occupe une «position de tout premier plan parmi les poètes latins au XVII ${ }^{\mathrm{e}}$ siècle». Il rivalise même avec Virgile (Voir M.-C. CHATELAIN, Ovide en France dans la seconde moitié $d u$ XVII siècle, Thèse de doctorat, Paris IV, juin 2005, p. 12. Voir également l'inventaire bibliographique dressé par l'auteur, pp. 615-631).

(68) Voir C. RizzA, La Métamorphose comme image et représentation du monde chez Théophile de Viau, in La Métamorphose dans la poésie baroque française et anglaise. Variations et résurgences, Actes du Colloque de Valenciennes, 1979, G. MathiEUCastellani éd., Tübingen-Paris, Narr-Place, 1980, pp. 135-142; R. Galli PellegrinI, Il linguaggio della metamorfosi nell'opera poetica di Théophile de Viau, in Metamorfosi, Mostri, Labirinti, G. CERINA et alii éd., Roma, Bulzoni, 1991, pp. 437-452; F. Schmidt Von Mühlenfels, Pyrame et Thisbé, in Pyramus und Thisbe. Rezeptions-typen eines Ovidischen Stoffes in Literatur, Kunst und Musik, Heidelberg, C. Winter, 1972.

(69) Pour ne citer qu'un exemple, l'humilité du «petit fonds» familial est un écho très certain des Élégies de Properce («Aspice me, cui parua domi fortuna relicta est...», II, 13).

(70) OC, cit., t. I, I, vv. 73-76, p. 123.

(71) Ibid., XVII, Ode, «Heureux tandis qu'il est vivant...», pp. 176-177. 
Il apparaît que l'amour des lettres antiques rencontre à cette date le goût du public. Les recueils collectifs en sont un témoignage assuré. Tout français qu'ils soient, les auteurs font voir, par le genre de la paraphrase poétique, les exigences d'une compétence latine. On continue d'y traduire les Psaumes, on fait d'Ovide l'incarnation de l'amoureux transi. Plus probant encore est le cas de l'édition de Théophile de Viau. Entre 1620 et 1621 paraît à Bordeaux un recueil de poésies de Théophile, disposé par l'éditeur comme une véritable scénographie de l'exil ovidien ${ }^{72}$. Des «tristes lieux» des «Landes de Castel-Ialoux» aux rives désolées du Pont, pour le Romain ou le mondain, les Gètes et les Gascons semblent rivaliser en barbarie ${ }^{73}$. L'auteur des Tristes et des Pontiques n'est pas seulement sollicité, dans l'écriture comme dans la disposition éditoriale des textes, pour arrimer les déboires de Théophile à ceux d'Ovide. La figure ovidienne se situe au croisement des intérêts de l'éditeur protestant G. Vernoy, rompu à l'édition des poètes latins, et du goût du public parlementaire bordelais à qui le recueil est dédié. De par son étendue, la culture latine est incontournable pour l'écrivain français, qu'il agisse en prosateur ou en poète. Elle autorise aussi, du point de vue de la publication, la constitution d'une figure de l'auteur. Comment celle-ci s'articule-t-elle au fait latin dans l'œuvre de Viau?

\subsection{Postures rhétoriques et jeux d'écriture, entre imitation et inventio}

Récuser l'imitation, préférer l'originalité, la critique à bon droit souligne la farouche volonté de Théophile de s'affranchir des modèles littéraires autant que des carcans sociaux. Pas plus qu'on ne saurait accorder d'autonomie réelle aux pratiques littéraires en ce premier XVII ${ }^{\mathrm{e}}$ siècle ${ }^{74}$, on ne saurait faire l'économie de la culture rhétorique de Viau. Lorsqu'il récuse les stériles imitateurs dans l'Élégie à Dame, n'oublions pas que le poète pratique là une forme d'imitation. La corneille d'Horace est là pour l'indiquer, l'art poétique théophilien de 1620 plonge ses racines dans une culture de l'anti-rhétorique ${ }^{75}$. À l'inverse, les réalisations en apparence imitatives n'excluent pas l'inventio. La paraphrase du Phédon en est un exemple achevé. Par le recours à la paraphrase, le traducteur semble s'en tenir à la définition de P. de Deimier ${ }^{76}$ : le texte français signale une fidélité constante à la version latine de J. de Serres depuis laquelle Théophile paraît entreprendre sa traduction. Mais les incursions versifiées procèdent d'une inventio savante ${ }^{77}$. C'est selon ce cadre esthétique qu'il faut envisager la pratique conjointe du latin et du français. Elle renforce l'idée selon laquelle Théophile parcourt sans cesse la gamme de l'imitation, entre attention scrupuleuse aux modèles anciens et audace inventive des genres.

Car, de l'imitation en apparence fidèle à la variation insensible, du détournement générique à la contamination des modèles, se décline une série de pratiques et de postures qu'il importe de recenser rapidement. Il peut s'agir de détourner le sens des emprunts ou des textes. Dans le Traicté par exemple, de simples amplifications

(72) On ne possède qu'une édition ultérieure, c'est le Nouveau recueil de diverses poésies du Sieur Théophile. La plus part, faictes durant son exil, Lyon, A. Soubron, 1622.

(73) Comparer à titre d'exemple $O C$, cit., t. I, LX, 1-8, p. 251 et Tristes, III, 3, vv. 5-14. Permettons-nous de signaler que notre doctorat dirigé par P. Ronzeaud - «Mon petit nom à l'ombre». Une bistoire de la figure de l'auteur dans l'auvre de Théophile de Viau (1619-1626) - consacre un chapitre à la constitution éditoriale d'une figure ovidienne de l'auteur.
(74) A. Viala, Naissance de l'écrivain, cit.

(75) G. Mathieu-Castellani, La rhétorique des passions, Paris, PUF, 2000, «Écriture», p. 117.

(76) L'Académie de l'art poétique, où par amples raisons, démonstrations, nouvelles recherches, examinations et authoritez d'exemples sont vivement esclaircis et deduicts les moyens par où l'on peut parvenir à la vraye et parfaicte connoissance de la poésie françoise, Paris, Jean de Bordeaulx, 1610, p. 255.

(77) Nous développons cette idée dans notre étude «Le Traicté de l'immortalité de l'âme...», ou les voix du traducteur, cit. 
contribuent à modifier considérablement l'appréciation apologétique du patron platonicien $^{78}$. Et la condamnation des plaisirs refusés au philosophe d'en devenir, par une série d'excursus poétiques, l'apologie discrète et ironique ${ }^{79}$. L'inventio dans un tel cas ne fait qu'accroître l'ambivalence idéologique qui pèse à cette date sur le corpus platonicien $^{80}$. Le détournement citationnel est souvent, dans la prose pamphlétaire, une manière d'adapter les patrons antiques à la situation de l'auteur. Plus fréquent encore est l'exercice de la contamination des modèles. Les cumuls citationnels sont légions. Contrepoints à la négligence en langue française, ils rappellent la solide érudition de l'auteur. La lecture, dans la Première journée, d'un billet de débauche de Sydias, écrit peut-être alors qu'il voit double, superpose à la citation enflée de Virgile («Eurusque Notusque ruunt» ${ }^{81}$ ) celle, plus sobre, de Sénèque, qui se l'était approprié ${ }^{82}$.

Quels sont les effets qu'induisent ces pratiques imitatives des textes latins? Elles permettent de déployer une «rhétorique du lecteur» (M. Charles). L'allusion est avant tout un moyen de confirmer la science du lecteur, qu'il soit parlementaire ou mondain. Il est des cas au contraire où elle dénigre la prétention au savoir. Quand la connaissance est infatuée, le recours est évidemment parodique: le latin de Sydias est «étourdi[ssant]», tant il sert à ses interlocuteurs proverbes éculés et paroles gelées de la scolastique: «j'ai passé [dit-il] la moitié de la nuit après cet intrigo de modalibus» ${ }^{83}$. Les paroles rapportées confirment la volonté de la narration de tourner en ridicule ce pot-pourri dissonant: «Sydias répondit [...] qu'il ne se promènerait point et que non poterat satis laudari Turcarum mos penes... $\gg^{84}$. Et dans cette satire, la référence aux compilations scolaires d'auteurs médiévaux entérine le discrédit jeté sur le pédant aveuglé qui entame une querelle sur les mots plutôt que sur les choses: «cet ignorant, dit-il, n'a jamais su les voix de Porphyre: O quam dura res est cum insipiente rem babere» ${ }^{85}$. Médiocrité dorée avant la lettre: il est bon de connaître le latin, il est bon surtout d'en avoir un usage discret et pertinent. Qu'il s'agisse de dénigrer un type de savoir, ou bien de le consacrer, le latin est un gage de connivence. L'assistance, dans la Première journée, s'amuse et «rit» des «conséquences» irrationnelles que tire de sa fréquentation frénétique des auteurs de la scolastique ce pédant «prêt à mourir sur [une] opinion» ${ }^{86}$. C'est grosso modo le sort réservé à Garasse dans l'Apologie. En rejouant les excès herméneutiques de la Doctrine curieuse, le locuteur rappelle que ce père-pédant en a perdu son latin:

Si le Père veut garder la signification du latin au français qui en dérive, il dira qu'une femme propre est la quatrième des cinq voix de Porphyre, et, ensuite de cela, une longue trâ-née d'absurdités qui se trouvent enchaînées dans les conséquences de ce Docteur ${ }^{87}$.

Renforçant l'ethos, ou lestant le pathos, comme dans la péroraison de l'Apologie, l'allusion latine a des vertus esthétiques: elle renforce l'hybridation générique dans la Première journée. L'entrée en scène de Sydias, entrée littéralement théâtrale ${ }^{88}$, inscrit la première déclaration du pédant dans l'univers comique d'un Plaute ou d'un Té-

(78) Ibid.

(79) Traicté de l'immortalité de l'âme, OC, cit., t. I, pp. 21-22.

(80) Voir J.-P. Cavaillé, Socrate libertin, in La Fortuna di Socrate nella cultura occidentale, Ettore Lojacono éd., Florence, Le Monnier, 2004.

(81) Énéide, I, 85.

(82) Quaestiones naturales, XVI, 2: «Uel, si breuius illos complecti mauis, in unam tempestatem, quod fieri nullo modo potest, congregentur: «una
Eurusque Notusque ruunt creberque procellis Africus» et, qui locum in illa rixa non habuit, Aquilo».

(83) Première journée, cit., p. 13.

(84) Ibid., p. 15.

(85) Ibid., p. 18.

(86) Ibid., pp. 18-19.

(87) Apologie de Théophile, cit., p. 173.

(88) «...m'approchant du lit de Sydias, comme je tirai son rideau, il s'éveilla en sursaut...», Première journée, cit., p. 13. 
rence: «Pro Deum atque bominum fidem» y est souvent une formule de maitre d'école ou de vieillard ${ }^{89}$. Et l'humour de relayer une critique efficace des excès libertins du noceur. C'est un Virgile moins grandiose que de coutume auquel fait référence Sydias lorsqu'il déclare:

\section{Nec veneris nec tu vini capiaris Amore...}

Un Virgile sans doute apocryphe qui ponctuait à la Renaissance corpus épigrammatiques et chansons à boire ${ }^{90}$. Un excès en engendrant un autre, la débauche de latin est le pendant d'une soûlographie gigantesque. De tels intertextes rappellent les connotations inattendues que supportent les auteurs antiques: le grand Virgile serait le père d'épigrammes; Platon, un Moïse parlant le langage attique, témoigne surtout, par la tranquillité de Socrate, de la méprise, sinon du mépris, de l'au-delà chrétien. Le régime de l'allusion signale surtout le jeu de connaissance que véhiculent, dans leur diversité, les pratiques imitatives de l'écriture latine.

\subsection{Une position linguistique de Viau? Du latin au français, idéal et nostalgie}

Par son latin macaronique, Sydias apparaît comme un contre-modèle rhétorique. Au discours des espèces et des catégories que supporte la diglossie du pédant, le narrateur oppose un style de digression. Cette figure en tout excessive légitime un renouvellement du style et de la langue, qui ne font qu'un, dès le chapitre liminaire de la Première journée:

Il faut que le discours soit ferme, que le sens soit naturel et facile, le langage exprès et signifiant; les afféteries ne sont que mollesse et artifice, qui ne se trouve jamais sans effort et sans confusion ${ }^{91}$.

L'unité du discours, du sens et du langage, recouvre, à la faveur toujours d'une comparaison entre le narrateur et le pédant, une différence notoire dans l'usage des facultés: à la mémoire figée du disputailleur, l'écrivain, dans ce premier chapitre toujours, fait le pari fructueux de l'imagination:

Ces digressions me plaisent, je me laisse aller à ma fantaisie, et, quelque pensée qui se présente, je n'en détourne point la plume... ${ }^{92}$

Cette série d'oppositions tend à placer la prose française du côté d'une valorisation sociale de l'idéal de la «conversation», quand le latin paraît affaire de «docte». Doit-on opposer le sens social de la langue française, à l'inadaptation constitutive d'un pédant qui, selon Clitiphon, quand «il croit avoir très bien rencontré» est «le plus orgueilleux pédant qui soit en son métier» ${ }^{93}$ ? La position linguistique de Viau

(89) L'expression se trouve certes dans les Catilinaires de Salluste (I, 20). Elle est surtout fréquente chez Plaute (Curculio, dans la bouche de Cappadox, V, 3, v. 693. Voir aussi Epidicus, IV, 2, v. 580) ou chez Térence (c'est l'injonction du vieillard Lachès dans L'Hécyre. Voir aussi l'Andria, I, 5, v. 246 ou encore l'Heautontimorumenos, I, 1). Les modèles renaissants ne sont pas en reste: c'est la formule du magister Eulubus dans le Poeticarum institutionum libri tres. Tyrocinium poeticum de J. Pontanus.

(90) L'épigramme est attribuée à Virgile par exemple dans le De generibus Ebriosorum et Ebrie- tate Vitanda, de 1515, réédité en 1617 et 1624 à Francfort (in Bacchi et Veneris facetiae..., I [BNF, RES- Y2- 2439]). Plus tard dans le siècle, Johann Michael Moscherosch dans ses Centuria considère toujours que Virgile en est l'auteur. J. Gallus en a tiré au XVI $\mathrm{e}^{\mathrm{e}}$ siècle une pièce musicale (Moralia, VI, Edo Skulj trans., Ljubljana, Slovenska akademija znanosti in umetnosti, «Monumenta artis musicae Sloveniae», 27, 1995).

(91) Première journée, cit., p. 11.

(92) Ibid., p. 12.

(93) Ibid., p. 15. 
est en réalité moins tranchée qu'il n'y paraît. C'est ce qu'indiquent les partis pris esthétiques du chapitre premier, où sont renvoyés dos à dos ceux qui, dans le sillage de Ronsard, goûtent abusivement «l'antiquaille», et ceux, esprits «faibles», «stériles» et pillards (entendez là les disciples de Malherbe), qui en marquent un «dégoût» trop affiché. Manque de «vigueur», carence de vocation et de savoir, c'est ce que semble vouloir conjurer le locuteur qui dans la fréquentation de Sydias ne manque pas de rappeler sa compétence latine. La contradiction ne se trouve donc pas là où il semble. Au-delà de l'exploration topique de la figure du pédant, Viau entend fonder une politique de la langue où le latin n'a pas seulement un rôle négatif. Manière d'essai par la forme, le vocabulaire et l'esprit, la Première journée entend transmuer la nostalgie du latin que véhiculent les Essais en idéal d'éloquence.

Car il existe bel et bien un envers à la caricature du latin dans l'œuvre de Viau. Elle est fondée par l'intertexte montaignien. Dans le même envoi de 1623 , le poète s'exclame, mélancolique:

Je n'ai qu'un pauvre fonds d'un médiocre esprit,

Où je vais cultiver ce que le Ciel m'apprit;

De tristes sons rimeurs, d'un style qui se traîne,

Épuisent tous les jours ma languissante veine.

Si j'avais la vigueur de ces fameux Latins,

Ou l'esprit de celui qui força les destins,

Qui vit à ses chansons les Parques désarmées

Et de tous les damnés les tortures charmées

Quand pour l'amour de lui le Prince des Enfers

Laissa vivre Eurydice et la tira des fers... ${ }^{94}$

La posture n'est pas seulement celle de l'bumilitas, qui sied à l'élégie. La faiblesse sociale d'un «petit nom à l'ombre», le désarroi humoral de l'élégiaque, la langueur «d'un style qui se traîne» et le manque d'éclat de «tristes sons rimeurs», en un mot le peu de vertus rhétoriques d'une plume appauvrie en «charmes», tout concourt certainement dans l'économie de l'élégie, comme par un effet d'ombre initial, à faire jaillir les «soins violents» (v. 25) de la passion qu'il va s'agir de décrire. Mais que le dispositif s'organise à partir d'un échec rappelle combien les postures de l'énonciation dépendent d'une carence constitutive de la langue, comme l'analysait Montaigne dans Sur des vers de Virgile. Impératif de vitalité dans la prose énergique de la Première journée, ou aveu d'impuissance dans la rumination élégiaque, la vigueur y occupe une place centrale. À partir de la lecture de Montaigne, Théophile se fixe trois objectifs et, en premier lieu, l'exigence d'un langage de «vigueur» venant compenser la faiblesse de la langue: «magnificence des anciens grecs et Latins» ${ }^{95}$ pour Montaigne également, car

À ces bonnes gens, il ne falloit d'aiguë et subtile rencontre: Leur langage est tout plein, et gros d'une vigueur naturelle et constante: Ils sont tout epigramme: non la queue seulement, mais la teste, l'estomach, et les pieds. Il n'y a rien d'efforcé, rien de trainant: tout y marche d'une pareille teneur. Contextus totus virilis est, non sunt circa flosculos occupatit ${ }^{96}$.

(94) OC, cit., t. II, Élégie, II, p. 33, on souligne.

(95) Première journée, cit., p. 11.

(96) Montaigne, Les Essais, cit., III, V, Sur des vers de Virgile, pp. 915-916. Les citations qui suivent sont tirées de ce passage. 
En premier lieu, le langage devra être «ferme», aussi «exprès» qu'expressif:

Ce n'est pas une eloquence molle, et seulement sans offence: elle est nerveuse et solide, qui ne plaist pas tant, comme elle remplit et ravit: et ravit le plus, les plus forts esprits.

Le pouvoir de ravissement proprement sexuel de la langue ${ }^{97}$ se réalisera, second objectif, dans une adéquation des mots aux choses. En valorisant l'imagination, la préséance de l'esprit sur le verbe est entérinée:

Quand je voy ces braves formes de s'expliquer, si vifves, si profondes, je ne dis pas que c'est bien dire, je dis que c'est bien penser. C'est la gaillardise de l'imagination, qui esleve et enfle les parolles. Pectus est quod disertum facit.

Toute la «rhétorique des passions» de Théophile semble ainsi se fonder sur ce lieu nostalgique de la langue latine. Elle se donne pour objectif final la représentation littéralement passionnée des êtres. Celui qui parle se fond en son objet:

Nos gens appellent jugement, langage, et beaux mots, les pleines conceptions. Cette peinture est conduitte, non tant par dexterité de la main, comme pour avoir l'objet plus vifvement empreint en l'ame. sions:

Tel semble être le désir d'enargeia de l'auteur, devenu le peintre de nos pas-

Peintre, n'as-tu rien fait encore?

Tu cherches trop de façon:

Il ne faut que peindre l'Aurore

Sous l'habit d'un jeune garçon ${ }^{98}$.

Tel est dans la prose et dans les vers, le vœu de vigueur dont hérite la langue théophilienne. Luttant fermement contre la mélancolie des «esprits finissants», la veine poétique s'animera (137). Un génie «abondant et prompt» (194), plein d'ardeur, d'éclat (120) et de vie (115), fera la peinture d'un «objet violent» (105). Faire «rajeunir le monde» (228), tel semble être le désir de la langue dans lequel «se coule» l'énonciation:

Comme si j'avais part à la faveur des Cieux,

Qui redonne l'enfance à ces bocages vieux,

Et que ce renouveau qui rend tout agréable,

Me rendit à tes yeux plus jeune et plus aimable ${ }^{99}$.

La conception linguistique chez Viau s'inspire donc d'une lecture des Essais. Idéal en acte dans la Première journée, dynamique nostalgique pour recouvrer dans l'élégie une langue perdue, c'est dans les deux cas un désir brûlant de parole vive qui devient, dans les essais de la prose latine, idéal d'éloquence.

(97) «Le latin serait [chez Montaigne] l'ultime refuge de la pudeur» selon T. CAvE, Cornucopia: $f$ gures de l'abondance au XVI siècle, G. MoreL trad., Paris, Macula, 1997 [1979], p. 290.
(98) OC, cit., t. II, 22, Thisbé pour le portrait de Pyrame/Au peintre, vv. 17-20, p. 74.

(99) Ibid., 2, Élégie, vv. 229-232, p. 39. 


\section{Incidence du latin dans la constitution d'une figure de l'auteur: la «latinité» de Théophile}

S'il ne fait pas de doute que la constitution progressive du champ littéraire oblige que l'on prenne acte d'une opposition entre les deux langues, Viau n'entend ni s'affranchir de l'une par l'autre, ni faire du français la pâle copie du latin. Saisi selon une continuité éditoriale, sociale et linguistique, l'emploi simultané des deux langues $^{100}$, à différents moments de l'œuvre, signale l'existence d'un idéal esthétique qui subsume, autant qu'il s'en nourrit, la divergence nostalgique de deux idiomes que rien ne réunira plus.

Car ce n'est pas un simple jeu de miroitement des langues auquel invite par exemple la comparaison du Theophilus et de l'Apologie. En voulant d'abord donner le change à Garasse et fonder une légitimité à l'écrivain contesté, l'incorporation d'une langue dans une autre permet de renforcer le prestige croissant de la poésie: importe moins le jugement moral (la condamnable paternité d'un texte manuscrit) que l'appréciation esthétique de la poésie. Importent davantage les censures et les licences linguistiques de «ce pauvre rimeur» ${ }^{101}$, plutôt que les torsions sémantiques de Garasse. La langue est la norme d'un jugement littéraire.

En prenant acte d'un écart entre les deux langues, l'auteur tente de conjurer l'écartèlement de sa situation. Comment à travers la réinvention continue de la prose latine Théophile parvient-il à fonder, en invoquant un idéal nostalgique du latin, une grandeur à sa figure d'auteur? Pour le dire autrement, il faut s'attacher enfin à montrer que le fait latin - langue, culture et écriture confondues - est l'une des figures constitutives de l'auctorialité théophilienne.

Nuançons d'emblée: les impératifs d'un style vigoureux et nerveux dans la prose française semblent éloignés du primat esthétique accordé à la douceur, de Larissa aux lettres latines. Insistant sur la «pureté» et «l'élégance» de la missive de son destinataire («quam terse et emendate scribas» ${ }^{102}$ ), le locuteur d'une lettre isole néanmoins une spécificité en acte de son tempérament de prose («Mibi vero si quid genius indulserat nascenti...> $\left.{ }^{103}\right)$. L'écriture latine recouvre en effet bon nombre d'options envisagées en français. D'abord, dans la relation mondaine des lettres comme dans les miscellanées de la Première journée, le primat est accordé au récit de soi. Seul semble varier le ton: il est voluptueux dans Larissa, badin dans la Première journée ${ }^{104}$. Il est souvent désolé dans les lettres latines, tant les aventures de l'exilé, aussi injustes soient-elles, mêlent tragique et ridicule ${ }^{105}$. La posture de l'exilé confine parfois à l'indifférence stö̈cienne ${ }^{106}$; elle peut instruire au contraire dans Larissa la tempérance d'un «maniérisme» noble et d'une «familiarité joviale» ${ }^{107}$; elle participe d'une rhétorique émouvante dans les lettres. Le locuteur dit son talent poétique «étouffé» par ses infortunes («Mibi si quid genius indulserat nascenti, pertinax adversae fortunae meae livor aut eripuit aut sepeli-

(100) R. Zuber parle en effet de complémentarité des deux langues dans la prose d'art (Les Émerveillements de la raison: classicismes littéraires du XVII siècle français, Paris, Klincksieck, 1997, «Théorie et critique à l'âge classique», p. 102).

(101) Apologie, p. 176, et Theophilus, p. 166. Dans les deux textes, le fameux vers français de l'Élégie à Tircis est mis face à des citations de Juvénal ou Virgile.

(102) OC, cit., t. III, Epistola VIII, p. 100.

(103) Ibid.

(104) «[...] faites-moi le conte de cette aventure [...] puisque vous avez la réputation d'être exacte- ment véritable jusqu'aux moindres choses, apprenez-moi comment tout s'est passé $[\ldots ..] \gg$, Première journée, cit., p. 16.

(105) Voir Epistola XVII: «Contigit mibi iter meum pergenti ridiculum quiddam, cujus te ut meoum omnium participem facere non erubescam», p. 105. 14.

(106) Première journée, cit., chapitre II, p. 13-

(107) L. Desjardin, «Larissa...», cit., rappelle que, dans l'esprit d'Ovide, le style noble de Larissa n'exclut pas les «expressions ingénieuses», et l'effet «violent» des mots et des images, p. 473. 
vit»). Le style en est la réalisation, à travers la construction hypothétique: «Si quando vacat aut licet de nostris aerumnis conqueri, habebitis ingenti volumine totam vitae meae syntaxim explanatam» ${ }^{108}$. Dans cette même lettre, la tension entre promesse épique («babebitis ingenti volumine totam vitae meae syntaxim explanatam») et faiblesse du locuteur devient, d'une phrase à l'autre, fait de style. Cela n'est plus douceur du commerce épistolaire, mais rupture pathétique, et accumulation malheureuse d'une prose où se télescopent passé et présent. Au moment où il envisage de faire la somme de sa vie, le locuteur confesse que la souffrance actuelle l'empêche de juger les anciennes passions: «Interim nova identidem infortunia de praeteritis nec gemere nec meditari sustinent ${ }^{109}$. Comme si la plénitude de l'être moral dans le récit en prose de la Première journée n'était que l'envers de cette dépossession de soi qui s'écrit dans la révélation érotique de Larissa, s'obscurcit dans la détention du Theophilus, pour finalement s'abriter dans l'intimité des lettres.

Comment la pratique du latin devient-elle, à travers diverses réalisations génériques, le creuset d'une invention éthique et esthétique de l'écriture en prose dans les années 1620? Appliquons-nous ici à en saisir les jalons.

\subsection{Larissa, tentation idéologique ou légitimité éthique de l'auteur imprimé?}

L'idéal latin s'interprète d'abord chez Viau comme une tentation idéologique. À travers le récit nostalgique d'une passion amoureuse, Larissa semble faire du latin la langue de la dissimulation. L'imaginaire «impubliable» d'un latin priapique, dans le sillage de Montaigne ${ }^{110}$, prédomine dans la lecture de Larissa, et cela bien au-delà du premier XVII siècle. Les traductions ultérieures s'appliqueront à censurer certains passages jugés indécents ${ }^{111}$. Doit-on cependant faire du latin «un élément supplémentaire de libertinage» ${ }^{12}$ ? À s'en tenir à la lettre du texte, il n'existe pas de passage réellement scabreux. À la différence de la production satyrique en langue française, les contempteurs de Théophile n'auront pas de prise sur ce texte. Est-ce en raison de la seule force dissimulatoire du latin? En réalité, la narration, héritière de la $\mathrm{co}^{-}$ pia verbale d'un Pétrone, s'interrompt au moment fatidique: «Quid plura?» ${ }^{113}$. Nous sommes loin des réalités crues du corps (le recours à la langue latine l'autorise, dans le corpus médical par exemple $\left.{ }^{114}\right)$. Le latin ne sert pas tant à masquer l'épicurisme qu'il ne participe à la construction d'une énonciation de la dissimulation. Par ce conte badin et folâtre (lascivus sermo), Théophile fait grincer des dents ceux qui condamnent Pétrone ou Apulée ${ }^{115}$. Il s'agit de construire un interdit. Tel est le sens, au moment où le récit de Larissa est suspendu, de l'intervention de «deux jeunes filles»:

Illae autem, inadversione simulata, ne sermoni castis animis refugiendo inverecundius interesse viderentur, faciem ab ore narrantis averterant, ac ingiter oscitantes, tum conniventibus oculis, nutanteque capite, molliter in somnum tota corporis specie fluere videbantur, ut quietis desiderium ementiae, tuto silentio indulgerent secretae libidini ${ }^{116}$.

(108) Epistola VIII, p. 100.

(109) Idem.

(110) T. Cave, Cornucopia, cit., p. 290.

(111) Voir par exemple le «dénouement pudiquement escamoté» de Larissa dans le Glaneur français de 1736, L. DESJARDIN, art. cit., p. 483.

(112) Fr. Waquet, Le Latin ou l'empire d'un signe, cit., p. 301.

(113) Larissa, p. 270. Voir par exemple dans le Satiricon la surenchère qu'autorise le «Quid multa?», cit., LXXVI, p. 142.

(114) Sur le latin, «langue des choses défendues», voir la synthèse de Fr. WAQUET, ibid., pp. 289-292 et J.-C. Abramovici, Obscénité et classicisme, Paris, PUF, 2003, «Perspectives littéraires».

(115) Scaliger s'était opposé en son temps à Scioppius, qui l'accusait d'avoir lu Pétrone par plaisir. La moralité de Pétrone et d'Apulée est discutée dès le début du XVII siècle (I. DE SMET, Innocence lost, or the implications of reading and writing (neolatin) prose fiction, in Eros et Priapus, érotisme et obscénité dans la littérature néo-latine, I. DE SMET et P. Ford éd., Genève, Droz, 1997, «Cahiers d'humanisme et Renaissance, 51», pp. 92-94).

(116) Larissa, p. 268. 
Ayant pris acte de leur jeune âge, la narratrice fait la promesse d'adoucir ses propos:

Puellis vero caeter se quam pudicissime posset absoluturam anus pollicita est ${ }^{117}$.

Mais les témoins de la scène ne sont pas dupes:

Tum his verbis tanquam data venia moribus improbis et quidvis audienti facta copia, virgines haud gravatim morem gerunt ${ }^{118}$.

Loin d'éloigner les oreilles trop chastes, l'introduction de ces deux personnages contribue à renforcer la curiosité sensuelle de l'auditoire pour ce conte délicieux («omnium aures ad sedulam attentionem erexerat»). Fortune de Pétrone... ${ }^{119}$. Car tout est fait dans ce texte pour accroître la tension entre une attente érotique de la lecture et les stratégies de rétention de l'évocation proprement sexuelle. Est ainsi mise au centre de la prose la tentation du lecteur, tant le texte s'organise selon la perspective d'un dévoilement progressif de la passion. Mais l'essentiel est contenu: «Nos deinceps libere clandestinis amoribus indulsimus» ${ }^{120}$.

Certes, le texte secrète des motifs épicuriens, comme autant d'indices d'une éthique des passions. Mais ces motifs sont relégués dans la fiction. La discrétion d'une narratrice qui n'a plus l'âge d'aimer n'estompe que faiblement ce «trésor de pudeur» qu'en sa jeunesse elle ne put contenir: «... quumque provectae libidinis ferociores essent impetus quam ut ulterius cobiberi possent...» ${ }^{121}$.

Le langage classique de la contamination amoureuse est débordé par la fureur de l'amour «... [qui] bouillonn[e] au plus profond des entrailles» ${ }^{122}$. La «crainte» n'est plus dans les paroles téméraires de l'aveu amoureux, mais dans l'angoisse d'un refus. L'évocation d'une toute puissance du corps sur l'esprit appartient au langage métaphorique d'une passion où l'âme se meurt:

Itaque desperandum fuit; quippe in tabescente corpore moriens anima suam sibi sepulturam foderat, ni misericordia fatorum meus amator conclamatae propemodum vitae meae salutis viam aperuisset ${ }^{123}$.

Surtout, l'énonciation, profitant de l'effet de décalage entre les voix narratives de Larissa et de l'auditoire, fait d'une injonction épicurienne ( $«$ Vos, dum per aetatem licet, vivite» ${ }^{124}$ ) fortement médiatisé par le Satiricon ( $\left\langle V i\right.$ is tu reuiuiscere ${ }^{125}$ ) un motif de la fiction, partagée entre le récit nostalgique de la vieillesse et l'évocation de la jeunesse vigoureuse. L'énonciation à proprement parler se substitue à la description de l'acte érotique: nous ne saurons rien de plus que ces longs baisers qui raniment le temps passé et ramènent au présent érotique. Car la nostalgie est comme un voile de gaze: «O diem nunquam rediturae voluptatis» ${ }^{126}$. La langue s'accorde à l'énonciation. Elle n'organise pas une transgression du texte, mais une liberté à la lecture. L'idéal du plaisir ne perce pas à jour le corps, mais, dans un jeu de chassé-croisé entre auteur et énonciation, elle construit une fiction du plaisir érotique, soutenu à cette date par

(117) Ibid.,p. 269.

(118) Idem.

(119) En marge par exemple d'un récit enchâssé: au moment où le narrateur s'apprête à raconter «une histoire vécue dont il avait gardé la mémoire», («Conversis igitur omnium in se uultibus auribusque sic orsus est», Satiricon, cit., pp. 218-219).
(120) Ibid., p. 270.

(121) Idem.

(122) Idem, trad. L. Desjardin.

(123) Idem.

(124) Satiricon, cit., p. 271.

(125) Ibid., p. 222.

(126) Larissa, p. 270. 
le modèle de l'imagination. Les égarements de la pudor qu'assume Larissa, à la fois personnage et narratrice, entre la délicatesse des mouvements pudiques du récit et ses résistances au plaisir, sont incarnés par les mots latins qui frappent l'imagination. Viau se pose ici en héritier d'une tradition d'écriture de la présence en langue latine. Superposant le temps du récit et le temps de l'histoire, l'énonciation latine entend sous nos yeux revivifier la beauté:

...enituit vultus pristino splendori restitutus tanta pulchritudine, ut Venerem referre potuisset eam quam Apelles dicitur effinxisse ${ }^{127}$.

Faire mention du peintre est très certainement une façon, encore, de souligner la dette envers Pétrone. Il s'agit surtout de renouer avec l'idéal d'une éloquence énergique.

Le recours au latin permet simultanément de conjurer un danger idéologique. La superposition des voix énonciatives, comme dans le Traicté, valorise une lecture hétérodoxe. Elle tente aussi d'innocenter l'auteur, dont la production est partagée à cette date entre reconnaissance mondaine et déni idéologique. Alliant la pudor de la langue à celle de la prose, et le sujet érotique à son expression contenue, Larissa représente une tentative de contrôle, dans le sillage de l'épître au lecteur ${ }^{128}$, d'une intégrité éthique de l'auteur. Cet équilibre, certes précaire, entre les incursions du libertinage et la conquête difficile d'une identité d'auteur, fait de la langue latine une garantie énonciative.

\subsection{La conquête d'une dignité: le Theophilus in carcere (1624)}

Lorsque, quelques années plus tard, Théophile renoue avec la prose latine dans le Theophilus in carcere, les vertus dissimulatoires de celle-ci servent un dessein très différent. La langue facilite certes, dans l'espace polémique, l'opposition entre une image mensongère de Théophile et la vérité d'une réputation de l'auteur:

Illa [La renommée] enim tam ficti quam veri nuntia, ego vero caetera pravus illud certe veracem esse me et intemeratae fidei nemo qui me novit diffitetur ${ }^{129}$.

Théophile rappelle à ses juges qu'il a été entraîné par «la rage de dénigrer» de son adversaire qui l'enveloppe entièrement de ses médisances:

Mirum nescire illum nocendi artem, cui noctes diesque insudet, in meam famam iam a suis primordiis imperitae turbae nebulonibus invisam ${ }^{130}$.

Théophile est-il tenté de répondre à tant de calomnies? Son intégrité de chrétien, plus encore que la faiblesse de sa situation, l'en empêche. Ainsi il n'optera pas pour la loi du talion ni pour l'opposition frontale à laquelle le style même du Theophilus semble un moment tenté:

Non licet bic nobis clavum clavo pellere aut conviciantibus conviciari ${ }^{131}$.

(127) Larissa, pp. 269-270.

(128) «[Je] veux sortir sans masque devant les plus rigoureux censeurs des écoles les plus chrétiennes. Je ne sache ni latin ni français, ni vers ni prose qui redoute la presse ni la lecture des plus délicats», Épître au lecteur, OC, cit., t. I, p. 6.

(129) Theophilus in carcere, cit., p. 162.

(130) Ibid., p. 163.

(131) Idem. 
Les atrocités de Garasse en langue française ne feront pas rougir ceux de sa compagnie. Par le choix du latin, Théophile sauvera les mœurs. Il se verra surtout permis de quitter un terrain proprement polémique:

Apage, scelus homine Christiano indignum, imo et dum mea se tutatur innocentia, ne tuus eror cuivis pateat, nolui vernaculo sermone tuas ineptias prodere ignavae plebi, cui tu tantum studes... ${ }^{132}$

À l'époque où Garasse se voit accusé de diffuser en langue vulgaire l'immoralité qu'il condamnait, le latin apparaît comme une langue immunitaire. Après Ogier, Théophile devient le censeur des impudicités du jésuite. Il s'agit d'abord de transcender l'injustice de la «langue scélérate» d'un Garasse:

Tua me impulit insania ut sane loquerer tua me adigunt mendacia ut vera dicam.

Aux «flots de médisances» (maledicendi licentia) nourries par la rhétorique plébéienne de ce Garassus imprudens, s'opposent les inflexions mesurées du prosateur, chez qui langue, érudition et sujet prennent l'apparence d'une même émotion rhétorique:

O insana turba, ignavum vulgus, vagi fluctus, caeci turbines, o vappa, o spuma rerum, virtutis inimica impotens, o rerum spuma vitiorum arca, o clamosa turba, invidiae tutissimum Praesidium, fidissimum calumnia subsidium, o foeda turba Garassi praecipuum decus, ignara nugarum vindex ${ }^{133}$.

Et Théophile, au faîte de sa période, de donner une véritable leçon d'innutrition à ce nouveau Sydias qui a «parlé sans réfléchir»:

Caeca turba cui nullum nomen nisi:

Fama malum quo non aliud, etc.

[Et] Tam ficit pravique, etc. ${ }^{134}$

En déplaçant la parole sur un forum imaginaire, Théophile va construire un ethos de vérité. Il y a de l'Apulée dans la diversité des ressources convoquées ${ }^{135}$, depuis la surenchère pathétique d'un locuteur qui, depuis sa prison obscure, veut capter l'auditoire, jusqu'au portrait bouffon du jésuite menteur; de l'interpellation régulière de l'adversaire par la question oratoire jusqu'à l'intégration d'excursus narratifs qui prouvent, comme autant de «faits», le repentir et la foi chrétienne de l'orateur ${ }^{136}$. La variété rhétorique ne tend qu'à prouver aux juges la justesse de la langue adoptée: elle est l'écho de leur justice. Puisqu'il ne convient pas de les flatter, il faudra leur parler le langage de la vérité et de la mesure cicéronienne, quitte à susciter les larmes des spectateurs:

Istic autem quidquid videris borridum, quidquid calcaveris sordidum, quidquid attigeris asperum, quidquid commederis factidum, quidquid biberis gelidum est... ${ }^{137}$

(132) Idem.

(133) Ibid., p. 167.

(134) Idem.

(135) Voir C. RizzA, L'art de Théophile de Viau polémiste, cit.

(136) Theophilus, cit., pp. 164-165.

(137) Ibid., p. 161. 
L'épreuve insoutenable de l'incarcération renvoie au caractère indiscutable de la loi: la prose, dans ses énumérations résistant à tous les assauts, se fait le relais d'une justice «implacable», à l'image des murs infranchissables de la Conciergerie:

Dura ligna, surdos lapides, rauca ferra nullis rimulis cuispiam aut oculis aut auribus aperta, nulla querela flectas, nulla arte fallas, nulla vi frangas; ipsum puto lovem incassum per baec invia aureos suos imbres emissurum... ${ }^{138}$

Il n'est plus l'heure de faire l'apologie de la liberté et des plaisirs par le latin. En défendant l'innocence d'une victime, le pathos s'intensifie. Si Glison s'était peu à peu guéri des dégoûts de sa captivité dans Larissa, le taedium, tout sénéquien, semble irrévocable pour le locuteur dont l'imagination même est dans les fers ${ }^{139}$. S'écrivent des passions subies et des passions dominées, des passions consenties et des passions dénigrées, s'invente en une prose vigoureuse une figure de l'auteur qui allie ethos et pathos. Le latin ouvre la voie dans le Theophilus à la reconquête d'une dignitas de l'auteur, idéal éthique et esthétique que les lettres latines vont consacrer.

\subsection{L'idéal éthique des lettres latines, ou l'invention de la «latinité» théophilienne}

L'univers épistolaire rompt assurément avec l'éloquence publique de la prose carcérale. Pourtant, la figure auctoriale reste au devant de la scène. Les ressources rhétoriques sont en apparence proches, tant le pathos contribue encore à marquer le sort particulier de l'auteur Théophile. Du strict point de vue chronologique, bon nombre de lettres continuent, sur le ton de la confession privée, ce que le Theophilus explorait sur la scène publique: dans le post-scriptum d'une lettre, Théophile avoue sa difficulté à reconquérir sa légendaire gaieté, tant la crainte des fers parait insurmontable:

Sed coram hujusmodi testibus qui judicum saltem imaginem ferunt si personam non sustinent, vixdum bene superati periculi extinctique rogi memor animus in sales et jocos excurrere libere aut frontem curis solutam explicare non audet ${ }^{i+1}$.

L'imagination semble marquée à jamais par les fers. Le présent est contaminé par les douleurs encore vives d'une captivité passée. La liberté a un goût amer:

Quid ergo mirum si quod praeteritae captivitatis amaritudinem auget et praesentis libertatis dulcedinem minuit etiam inter epulas aversetur aut impatienter ferat ${ }^{14}$ ?

Ce n'est plus la prison, mais l'exil qui fournit matière à une prose devenue chagrine. Parfois, l'absence s'annonce aussi triste que celle d'Ovide: $d a e^{142}$

Quippe nobis assignatur apud oceanum vaga et pericolusa sedes: scopuli, vada, vetus et un-

Elle n'est ailleurs que l'évocation badine d'une situation publique de l'auteur Théophile. Faisant l'éloge de l'accueil du Comte de Béthune, il déclare à Pitard:

(138) Ibid., p. 162.

(139) Theophilus, pp. 161-162. Comparer Larissa, cit., «Gliso iampridem a fastidio veteris tristitiae liberior...», p. 270; Theophilus, «diutissimae servi- tutis taedia», p. 161.

(140) Epistola IV. Ad dominum Lulerium, p. 97.

(141) Ibid., pp. 97-98.

(142) Epistola XVI. Ad Vallaeum, p. 105. 
Omnia denique hic bonae mentis oblectamenta nobis suppetunt, ut plane intelligas Theophilum paulo quam Nasonem suavius exulass $e^{143}$.

L'auteur ne s'installe plus, comme en 1621, dans un rapport conflictuel avec les modèles antiques. S'il s'étonne que ses correspondants aient pensé à Sénèque en le lisant, c'est simplement en raison du peu de conformité entre leurs deux génies:

... revoca si possis in memoriam illum Senecae locum ex quo me plagiarium suspicamini: vix contigisse puto ut idem sensus in tam dissimiles inciderit animos ${ }^{144}$.

Fondées par le cadre privé de l'échange, repli social que le recours au latin conforte, les lettres favorisent l'écriture de Théophile par lui-même. Fréquemment il mentionne sa situation d'exilé et ses mésaventures. Doit-on considérer comme un simple projet, que la mort brutale du poète a empêché de faire aboutir, la promesse de Théophile à ses protecteurs: «habebis aliquando ingenti volumine explanatam rerum nostrarum seriem» (XXII)? S'il promettait encore à Candale de faire de ses malheurs, de ses infortunes, en un mot de sa vie, un ouvrage relevant davantage du genre historique qu'épistolaire («Tot insultus fortunae, tam varias vitae nostrae vices, Historiae est non Epistolae complecti〉), n'y a-t-il pas lieu de croire que les lettres latines en sont une manière d'essai, puisqu'elles sont le lieu où est figuré l'écart entre les desseins de l'écriture et les infortunes de la vie («babebitis ingenti volumine totam vitae meae syntaxim explanatam», VIII)? Les lettres latines font de Théophile la matière de sa prose.

Car elles sont bien le lieu d'une recherche de grandeur sociale et intellectuelle du dernier Théophile. Sous la plume latine de Viau, l'auteur apparâtt en une singulière, mais cohérente, figure de savoir. Le latin n'est pas seulement, comme pour beaucoup de ses contemporains, un instrument de communication intellectuelle ${ }^{145}$. Il propose une image homogène des postulats idéologiques de Théophile. Postulat éminemment critique tant il importe, en reconduisant le parallèle de la Première journée entre les «bavardages d'ivrognes» et le «vacarme de nos disputailleurs», de dénoncer les pensées brouillonnes de son temps ${ }^{146}$. Est ainsi développée une critique de la confusion:

At illi, quam se intricent, quam variis erroribus sibi caeterisque fucum faciant, quam incertis vaticiniis credulam hominum curiositatem et foveant et eludant videre est... ${ }^{147}$

Socrate, «ennemi du discours qui tente,/Et qui suborne les esprits» dans le Traicté de l'immortalité de l'âme, ne dit pas autre chose: «les contentieux [...] ne laissent point à faute de sapience de plaire et trouver leur compte dans cet embrouillement dont ils confondent tout» ${ }^{148}$. Et comme chez celui dont «l'humeur [est] toute contraire» aux misologues, il s'agira de rendre exemplaire la position intellectuelle de Théophile qui déclare, laconique, à son interlocuteur: «ego bactenus feci» ${ }^{149}$. Une telle critique formalise surtout l'humilité du sujet connaissant selon Théophile. Soit le locuteur s'applique à se distinguer de l'érudition proprement dite: «natura enim fecit eruditum», dit-il à propos de J. Vallée des Barreaux, dont le «genius» est contraire à celui de l'épistolier: «Mibi vero... ${ }^{150}$. Soit, à la faveur d'un échange avec un savant

(143) Epistola XIX. Ad Eumdem, p. 109.

(144) Epistola XII. Ad carissimum Vallaeum, p. 103 .

(145) Voir par exemple les lettres XVIII et XIX [adressées au] «doctissimum virum Pitardum».

(146) Epistola IX. Ad dominum Lulerium, p. 101.

(147) Idem.

(148) Traicté, cit., p. 80.

(149) Epistola IX, p.101.

(150) Epistola VIII. Ad dominum de la Pigeonnière, p. 100. 
de son temps, il prend la posture de l'ignorant ou du novice ${ }^{151}$. La grandeur du savoir réside surtout dans la prudence sceptique de Théophile:

Quid mea refert quid aut iste [un "novus author" imposteur] aut prisci omnes de mundi causa investigaverint, cum plane constet nibil illos de tanta re compertum unquam habuisse ${ }^{152}$ ?

L'anthropologie de la connaissance, telle qu'elle se dégage des lettres latines, est indissociable d'une quête morale. Elle exprime, force de l'bumilitas et de l'expérience, les progrès d'une foi récente: après avoir cité l'Énéide dans une perspective néo-testamentaire - à moins qu'il ne s'agisse d'une pointe («Neque haec sine numine divum/Eveniunt» ${ }^{153}$ ) -, le locuteur rappelle le chemin dans lequel il s'est engagé:

Caeterum magis magisque propagatur in nobis catholicae pietatis amor, et diebus singulis ad altaria et mentem et genua flectere jam cessit in voluptatem: uno verbo Theophilus sum ${ }^{154}$.

La connaissance réside dans la conquête de soi et dans la reconquête de son propre nom. Mais l'identité est à jamais insaisissable: l'espace couvert des lettres latines rend sensible, dans le nom même de l'auteur, un contact mystique avec soi-même. Car la volonté de connaître chez Théophile n'a de pertinence qu'en ce qu'elle fait les hommes meilleurs. Ainsi le bien-fondé d'une secte philosophique est contesté pour des raisons éthiques: $\operatorname{rim} \ldots$

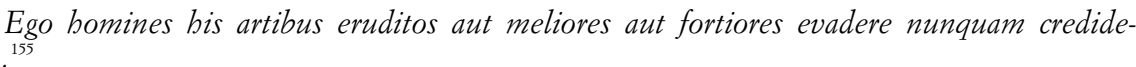

La latinitas exprime dans l'espace réservé des lettres latines l'ambition d'un discours caractérologique sur l'humain où se fondent «des affinités électives» ${ }^{156}$. S'étonnant que le tempérament lascif de Des Barreaux ne l'ait entièrement détourné d'une science ferme, Théophile déclare à La Pigeonnière:

Miror unde tantillum latinitatis in tam lubrico ingenio baerere possit ${ }^{157}$.

Cet idéal de connaissance de soi et de l'autre a un seul nom dans les lettres: l'amitié. Seuls quelques élus peuvent participer à cet espace de sociabilité latine dont la langue est le symbolum et dans laquelle l'énonciation se replie ${ }^{158}$. À la faveur d'un partage renouvelé entre otium et negotium ${ }^{159}$, l'amitié se dit dans des lieux de retraite, dans des jardins de délices ${ }^{160}$. Un langage la consacre, c'est celui où s'éprouve une culture entièrement partagée ${ }^{161}$; c'est celui où se reconquiert, dans le désir de la «vive voix» $(\mathrm{V})$, une «libera libertas».

(151) Epistola XIX, cit., «Consulo te de mei instituti ratione, et quibus potissimum philosophorum libris credere debeam initium laboris mei».

(152) Epistola IX, cit., p. 101.

(153) Enéide, II, 777-778.

(154) Epistola V. Ad Eumdem, p. 98.

(155) Epistola IX, cit., p. 101.

(156) G. SABA, Théophile de Viau: un poète rebelle, cit., p. 207.

(157) Epistola VIII, cit., p. 100.

(158) Voir par exemple le souci religieux de soi auquel il convie son frère: «Consule te ipsum», Epistola XXIV, p. 111; ou l'amicale expressivité de la langue latine: «Nibil habeo quod ad te scribam, at scribo tamen; tu quoque licet nullam habeas amandi mei causam, ama me tamen», Epistola XVI, cité par G. SABA, Théophile de Viau: un poète rebelle, cit., p. 209.

(159) Epistola V. Ad Eumdem, p. 98.

(160) Epistola XIX. Ad Eumdem, p. 108.

(161) Tel est par exemple l'effet énonciatif de la seconde lettre qui, répondant à la garantie d'amitié épique de J. Vallée Des Barreaux («Una quies ambobus erit», Epistola I. Vallaeus Theophilo suo, p. 95) par la feinte exaspération d'un Ovide $(\ll \mathrm{Nec}$ venit ante suum nostra querela diem», Hérö̈des, II, 8), fait de l'énonciation ludique sur la lettre et les langues un signe d'amitié: la médiation du texte 
Le fait latin participe de la profonde diversité qu'imprime l'auteur à son œuvre. Tout en recouvrant des usages et des acceptions multiples, le latin est le signe, chez Théophile de Viau, d'une culture et d'babitus humanistes pleinement assumés. La langue latine jette-t-elle une ombre sur la modernité de Théophile? Elle rend compte plutôt d'un dialogue fructueux entre l'ancien et le moderne, le docte et le mondain. À la nostalgie d'une vigueur toute latine dont l'élégie française porte le deuil fait écho la recherche d'une énergie renouvelée dans la prose, latine ou française. À l'ombre d'un Balzac, le choix de l'écriture latine contribue à un idéal d'éloquence personnelle, à l'heure où le prestige poétique serait sans effet s'il ne s'accompagnait de dignité sociale. Cette langue qui masquait le plaisir érotique pour mieux le faire transparầtre (Larissa), celle-là même qui voile les «ordures» que lui allèguent ses détracteurs (Theophilus), est celle aussi qui révèle les mensonges populaires (Première journée, Apologie de Théophile) et révère, comme une vérité rare, le secret de l'amitié. Dans la double évocation d'un idéal et d'une nostalgie du latin, Théophile de Viau fait de la langue antique l'instrument moderne d'une anthropologie de la connaissance. Mairet ne dira-t-il pas à juste titre de Théophile qu'il fut, à l'égal de Montaigne, un «Sénèque de notre âge et de notre langue» ${ }^{162}$ ?

MELAINE FOLLIARD

ovidien («nostra querela») métamorphose la solitude de l'exilé («fortuna mea», «injuria mea», «mea sortem») en connivence épistolaire («nostra epis- tola», «tua epistola»).

(162) Nouvelles Euvres, OC, cit., t. III, p. 10. 